\title{
„Präventionsforschung“ als Förderschwerpunkt des BMBF - Ein erfolgreicher Vorstoß in die richtige Richtung!
}

Prävention und Gesundheitsförderung sind in Deutschland geprägt von einer Vielzahl an Akteuren sowie zahlreichen, häufig nicht evaluierten Projekten. Forschung in der Primärprävention und Gesundheitsförderung (PGF) wird zunehmend seit den 1980er Jahren staatlich gefördert. Hierzu zählen insbesondere die gemeindebezogene Deutsche Herz-Kreislauf-Präventionsstudie sowie die Public Health-Forschung. Mit dem Förderschwerpunkt „Präventionsforschung“ richtete das Bundesministerium für Bildung und Forschung (BMBF) erstmals einen eigenständigen Förderbereich ein, in dem die PGF von 2004 bis 2012 - mit Auslaufen der letzten Projekte Anfang 2013 - explizit unterstützt wurde.

Erfahrungen anwendungsorientierter Forschungsförderungen zur Rehabilitations- und Versorgungsforschung zeigen, dass über ein Förderprogramm die Qualität und Wirksamkeit der Maßnahmen gesteigert, die Methodik verbessert und eine internationale Anschlussfähigkeit von Forschergruppen erreicht werden kann. Ähnlich wie die Rehabilitation ist auch die PGF geprägt von Inter- und Multidisziplinarität: Ihre erforderliche Integration in die Bereiche Bildung, $\mathrm{Ar}$ beit, Gesundheit und Soziales erfordert eine hohe Anschlussfähigkeit der Inhalte und Methoden. Allerdings ist die Ausgangslage hinsichtlich der Vielfalt der Akteure und der gesetzlichen Grundlagen deutlich heterogener und geprägt von unsicherer finanzieller Absicherung. Die Einrichtung des BMBF-Förderschwerpunkts „Präventionsforschung “ ist als notwendige Initiative zur nachhaltigen Strukturbildung der deutschen Präventionsforschung zu verstehen.

Der Schwerpunkt umfasste in allen 4 Förderphasen 60 Projekte. Eingebunden waren über 50 wissenschaftliche Einrichtungen und 170 Praxispartner, Akteure in den Bereichen Gesundheit, Bildung, Arbeit und Soziales auf kommunaler, Landes- und Bundesebene. Inhaltlich überprüften die Projekte die Entwicklung und Erprobung neuer Zugangswege, geeigneter Methodiken sowie die Akzeptanz und Wirksamkeit von Interventionen. Im letzten Drittel der Förderung wurde das Meta-Projekt „Kooperation für nachhaltige Präventionsforschung“ (KNP) initiiert. Hiermit wurde die Distribution wesentlicher Erkenntnisse unterstützt, die langfristige Vernetzung zwischen Wissenschaft, Praxis und Politik gefördert und Wissensmanagement im Bereich der Präventionsforschung aufgebaut. Das mehrdimensionale Vorgehen, aber auch die Grenzen eines solchen Vorhabens werden einleitend dargelegt. Das Supplement gibt einen Überblick über wesentliche Ergebnisse der Projekte des Förderschwer- punkts, die von den Beteiligten des Verbundprojektes, insbesondere der Medizinischen Hochschule Hannover (MHH), des Universitätsklinikums Hamburg-Eppendorf (UKE) und der Bundeszentrale für gesundheitliche Aufklärung (BZgA), im Rahmen von KNP zusammengeführt und in 5 inhaltliche Querschnittsbereiche gegliedert wurden:

- Zielgruppen erreichen - Zugangswege gestalten

- Kinder und Jugendliche - Präventive Maßnahmen wirken

- Von jungen Erwachsenen bis zum höheren Alter - Präventive Maßnahmen wirksam gestalten

- Strukturen ändern - Gesundheit integrieren

- Präventive Maßnahmen transferieren - In Versorgungspraxis überführen

Die denkbar größte Anerkennung für die von allen Beteiligten geleistete Arbeit im Förderschwerpunkt ist die seit 2014/2015 laufende BMBF-Förderung von „Verbünden zur Präventionsforschung“" sowie die erstmalige Förderung von Nacherhebungen zur Evaluation der Nachhaltigkeit in der Prävention und Gesundheitsförderung. Der Fortgang dieser Forschungsförderungen wurde seitens KNP explizit vorangetrieben. Die Entwicklung dreier Memoranden zur Forschungsförderung und Strukturbildung beeinflusste darüber hinaus auch die politische Debatte um das Präventionsgesetz. Zudem konnten einige von KNP mit aufgebaute thematische Arbeitsgruppen über die Integration in Fachgesellschaften verstetigt werden. Die vielerorts wertgeschätzte strukturbildende Arbeit von KNP, insbesondere zum kontinuierlichen Austausch zwischen Wissenschaft, Praxis und Politik, wird allerdings nicht weiter gefördert, sodass die in immenser Aufbauarbeit unter Einbezug zahlreicher Wissenschaftler/-innen und Akteure geschaffene Synergien und Know-How verloren gehen. KNP ist allerdings kein Einzelfall und verweist damit auf Probleme in der deutschen Förderlandschaft.

Die Zukunft der PGF in Deutschland bleibt dennoch spannend. Hier werden wesentlich die Erkenntnisse der Forschung, deren nachhaltige Umsetzung in die Praxis und der politische Wille entscheidend sein. Erwartungsvoll richten sich nun erst einmal die Blicke auf die Umsetzung des Präventionsgesetzes und die dort bevorstehende Ausgestaltung einer nationalen Präventionsstrategie. Die wissenschaftlichen Erkenntnisse des BMBF-Förderschwerpunktes Präventionsforschung können dazu einen Beitrag leisten.

Prof. Dr. Ulla Walter, Dr. Martina Plaumann, Dr. Silke Pawils, Dr. Guido Nöcker, Prof. Dr. Elisabeth Pott, Prof. Dr. Dr. Uwe Koch 